\title{
Bilateral counter movement jump, squat, and drop jump performances in deaf and normal-hearing volleyball players: a comparative study
}

\author{
Yücel Makaracı*, Ömer Özer, Recep Soslu, Abdullah Uysal \\ Faculty of Sports Sciences, Karamanoğlu Mehmetbey University, Karaman, Turkey
}

Since deaf athletes do not have any physical deficiencies, it is known that they can easily participate in sports competitions if their communication problems are resolved. This study aimed to compare the performances of olympic deaf and elite normal-hearing volleyball players in different jumping test protocols. A total of 26 male volleyball players participated in the study, comprised of 12 olympic level deaf and 14 elite level normal-hearing. Following anthropometric measurements, athletes performed bilateral counter movement, squat and drop jump tests on a force plate. Each test was performed 3 times with maximal effort separated by 45 seconds of passive recovery and the mean of the three trials was selected for analysis. Independent Samples $t$-test was used to compare the differences in mean values of jumping test parameters between the groups. Normal-hearing athletes are observed to have significantly better scores than deaf athletes in all test protocols. Considering that the number of studies on athletic performance in deaf athletes is limited, it can be said that our study will contribute to researchers and coaches in respect of detecting a key ability in volleyball, such as jumping, in different jump protocols.

Keywords: Explosive power, Hearing-impared, Jump, Lower extremity, Volleyball

\section{INTRODUCTION}

In volleyball, which is a team sport, abilities such as quick and explosive power come into prominence, particularly in the struggles for possession of the ball (Mroczek et al., 2019; Tramel et al., 2019). Optimum performance of the block and jump serve varies according to the explosive power output of the lower extremities. The vertical jump, which is a reflection of lower extremity explosive power, takes place frequently in rallies and other actions during the competition (Pérez-Turpin et al., 2014). Therefore, lower extremity explosive power in a vertical jump is one of the most important motor movements required for success in volleyball (Mroczek et al., 2017). This explosive movement should not only be interpreted from perspectives such as jumping height or power but also be performed with the right timing (Fuchs et al., 2019). The effect of jumping ability in volleyball on defensive and offensive ac-

tions can be explained by the contact of the ball with the opposing team's court, which is the main goal (Gonçalves et al., 2021).

Vertical jumps on the force platform enable the analysis of multijoint structures in terms of their similarity with the movements specific to volleyball (e.g., closed kinetic chain activities and stretch shortening cycle) (Schons et al., 2018). Furthermore, "acceleration," "force," and "impulse" values can be determined during jumping (Lake et al., 2018). Jump protocols taking place in the vertical axis are widely used to determine the jumping height and lower extremity strength (Borràs et al., 2011). Counter movement jump (CMJ), squat jump (SJ), and drop jump (DJ) have been the prominent methods in determining jumping capacity in recent years (Wong et al., 2020). In the biomechanically similar CMJ and SJ, the movement that begins with standing ends with a sudden jumping movement after a downward squat. While time-related factors arising from waiting in the semisquat position affect performance
*Corresponding author: Yücel Makarac1 (iD https://orcid.org/0000-0002-6891-9916 Faculty of Sport Sciences, Karamanoğlu Mehmetbey University, 70200 Karaman, Turkey

Email: yucelmkrc@gmail.com

Received: September 6, 2021 / Accepted: September 14, 2021
This is an Open Access article distributed under the terms of the Creative Commons Attribution Non-Commercial License (https://creativecommons.org/licenses/by-nc/4.0/) which permits unrestricted non-commercial use, distribution, and reproduction in any medium, provided the original work is properly cited. 
in SJ, time-related factors are excluded in CMJ (Van Hooren and Zolotarjova, 2017). Moreover, the determination of the CMJ, especially by using the force platform, provides very reliable data in terms of the evaluation of mechanical power (Garcia-Ramos et al., 2017). DJ, which takes place as an explosive jump after dropping from a certain height, is similar to block and repetitive jumps in volleyball in terms of movement mechanics (Peng et al., 2019). In jump protocols occurring in the vertical axis, for a drop in a predetermined area, the movement series that includes planning the route to be followed by the body mass center, controlling the body position during the jumping phase, and providing postural control with ground contact (landing) needs to be performed successfully (McKinley and Pedotti, 1992).

Although individuals with disabilities have some difficulties in participating in sports activities, the difficulties experienced are eliminated to a large extent owing to the sports branches specially designed according to the type of disability (Akınoğlu and Kocahan, 2019). Since deaf athletes do not have any physical deficiencies, it is known that they can easily participate in sports competitions if their communication problems are resolved (Kurková et al., 2011). In this context, the evaluation of jump (Gonçalves et al., 2021), one of the primary performance outputs for success in volleyball, in deaf volleyball players will constitute a reference in terms of interpreting the athletic performance levels of athletes. In the literature, the number of studies examining jump performance in deaf athletes is quite limited. The aim of this study is to compare the performances of deaf and normal-hearing volleyball players in different jump tests based on the significance of lower extremity explosive power in volleyball.

\section{MATERIALS AND METHODS}

\section{Study structure}

A purposive sampling method was used to investigate the jumping performance of deaf and normal-hearing volleyball players by using different protocols The study were conducted after the 2020 2021 Turkish Men's Volleyball Leagues. The tests were carried out at the training facilities of the athletes. The measurements of both groups were taken at the same time of day (mid-day). A sign language translator in the interaction with deaf players was present throughout the study to explain the test procedures. A separate familiarization session before the testing day was not deemed necessary, as this is not required with participants of this level (Psycharakis et al., 2019).

Following anthropometric measurements, a standardised warm- up preceded the jumping tests, which involved 5 min of jogging, 5 min of passive stretching and three maximal vertical jumps. The warm-up session was followed by a 5-min period of resting (Theodorou et al., 2013). After warm-up process, participants performed CMJ, SJ, and DJ tests on a force plate in a randomised order. Each test was performed 3 times with maximal effort separated by $45 \mathrm{sec}$ of passive recovery and the mean of the three trials were selected for analysis (Barker et al., 2018; Suarez-Arrones et al., 2020). The participants were instructed to jump as high as possible during all test protocols and to keep their hands on their hips. No other instructions were specified (Schons et al., 2018). Jumps not meeting these requirements were repeated. To reduce the interference of uncontrolled variables, all subjects were instructed to maintain their usual way of life and routine diet programme intake before and during the study.

\section{Participants}

A total of 26 male volleyball players participated in the study, comprised of 12 olympic level Turkish deaf volleyball team members within the Turkish Deaf Sport Association ( $\mathrm{n}=12$, mean \pm standard deviation [SD], age: $24.25 \pm 4.31$ years, height: $187.08 \pm$ $7.15 \mathrm{~cm}$, weight: $79.92 \pm 9.42 \mathrm{~kg}$, body mass index [BMI]: $22.43 \pm$ $\left.1.93 \mathrm{~kg} / \mathrm{m}^{2}\right)$ and 14 elite level normal-hearing $(\mathrm{n}=14$; mean $\pm \mathrm{SD}$, age: $22.07 \pm 3.20$ years, height: $195.29 \pm 4.30 \mathrm{~cm}$, weight: $85.64 \pm$ $8.87 \mathrm{~kg}$, BMI: $22.9 \pm 2.793 \mathrm{~kg} / \mathrm{m}^{2}$ ). The Turkish Deaf Sport Association is represented by the people who have hearing loss of at least at a moderate level of above $55 \mathrm{~dB}$.

The selection criteria of the athletes were; being older than 18 years old, being a volleyball player for at least 3 years, having a medically diagnosed hearing impairment condition/ability to understand basic instructions (for deaf athletes) and volunteering to participate in this study. The elimination criteria included: having a recent acute injury causing cognitive, physical or visual impairments (for deaf athletes) and having any neurological, orthopedic or cardiovascular diseases. Subjects were asked to avoid maximal physical exertion $48 \mathrm{hr}$ before testing. All subjects completed informed consent forms. Ethical approval (Document No: 26793/ 2021) was obtained from the Clinical Research Ethics Committee of a state university, in accordance with the Declaration of Helsinki.

\section{Anthropometric variables}

The athletes' body weight was measured by the force plate (automatically before jumping tests) and their height were measured by a stadiometer (SECA-Mod.220, Seca GmbH \& Co. KG., Hamburg, Germany). The formula used for calculating the BMI is tak- 
ing weight in kilograms $(\mathrm{kg})$ divided by height in meters $(\mathrm{m})$ squared.

\section{Test protocols}

A force plate (Kistler, type 5691A, Winterthur, Switzerland; $40 \mathrm{~cm} \times 60 \mathrm{~cm}$ ) with the sampling frequency of $1,000 \mathrm{~Hz}$ was used to obtain the vertical ground reaction force data on the pushoff and landing phases of the athletes in different jumping test protocols. Both legs were used during the push-off $\mathrm{f}$ and landing phases.

\section{CMJ test}

Participants performed bilateral CMJ test where both legs were used during the push-off phase. Test was performed at $60^{\circ}$ knee flexion angle. All jumps were performed with the hands on the hips (Pérez-Castilla et al., 2019) to minimize the influence of upper-body movements on center of mass location. Participants were instructed to perform a fast counter movement until reaching the predetermined knee flexion angle and to jump as high as possible.

\section{SJ test}

In the SJ test, the participants were instructed to stand, flex the knees to a semisquat position and jump. The participants had to avoid as much as possible any countermovement, and they were instructed to stop for 2 sec at each phase (Coratella et al., 2018). Three maximal SJ performances are recorded.

\section{DJ test}

For the DJ test, participants started by standing on a $30-\mathrm{cm}$ high box with feet shoulder-width apart. A target line was placed at a distance equal to half the body height of each participant away from the front of the box. Participants jumped forward just beyond the line. Upon landing, participants jumped vertically as high aspossible without restriction of arm movements (Beardt et al., 2018).

As a result of the jumping tests performed, relative maximal power (RMP), average power (AP), average force (AF), average velocity (AV), acceleration (ACL), jump height from take off velocity (JHTOV), vertical take of velocity (VTOV), and flight time (FT) parameters obtained from the Kistler measurement, analysis and reporting software (MARS) were used for the statistical analysis. Movement characteristics of each jumping protocol obtained from the MARS are presented in Fig. 1.

\section{Statistical analysis}

The statistical analyses were performed using IBM SPSS Statistics ver. 24.0 (IBM Co., Armonk, NY, USA). Descriptive statistics are reported as mean $(\overline{\mathrm{X}}), \mathrm{SD}$, and standard error of the mean values. The data were found to be normally distributed based on the Kolmogorov- Smirnov test and skewness/kurtosis values. Independent Samples $t$-test was used to compare the differences in mean CMJ, SJ, and DJ parameters between normal-hearing and deaf volleyball players, Significance for all comparisons was set a

\section{Bilateral Counter Movement Jump}

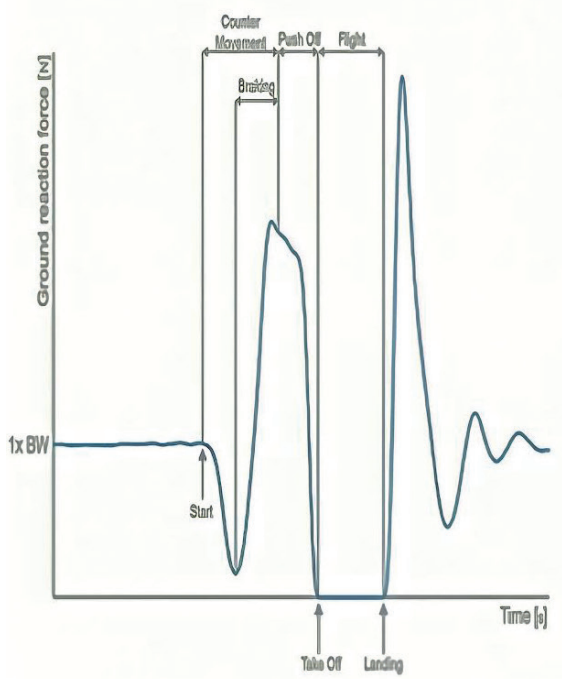

Squat Jump

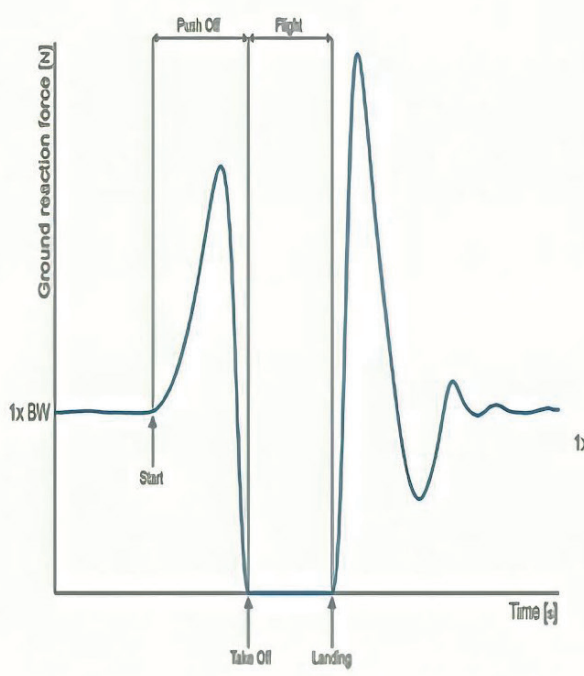

\section{Drop Jump}

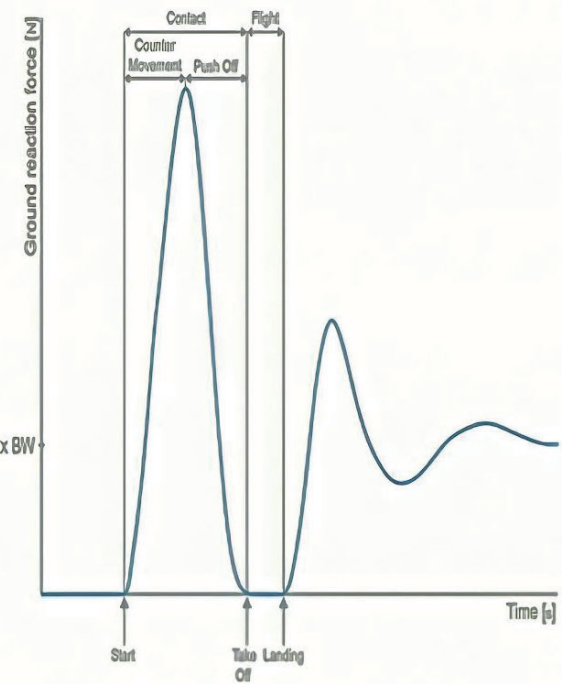

Fig. 1. Movement characteristics of CMJ, SJ, and DJ test obtained from the MARS. CMJ, counter movement jump; SJ, squat jump; DJ, drop jump, MARS, measurement, analysis and reporting software. 
Table 1. Comparasion of the athletes by counter movement jump test

\begin{tabular}{|c|c|c|c|c|c|c|c|c|}
\hline Parameter & Group & No. & $\bar{X}$ & SD & SEM & $t$ & $P$-value & Cohen $d^{t}$ \\
\hline \multirow[t]{2}{*}{ JHTOV (m) } & Normal-hearing & 14 & $0.41^{*}$ & 0.09 & 0.02 & 2.06 & 0.050 & 0.81 \\
\hline & Deaf & 12 & 0.33 & 0.10 & 0.02 & & & \\
\hline \multirow[t]{2}{*}{ RMP (W/kg) } & Normal-hearing & 14 & $57.68^{*}$ & 8.82 & 2.35 & 2.66 & 0.014 & 1.03 \\
\hline & Deaf & 12 & 48.94 & 7.78 & 2.24 & & & \\
\hline \multirow[t]{2}{*}{$\mathrm{ACL}\left(\mathrm{m} / \mathrm{sec}^{2}\right)$} & Normal-hearing & 14 & 3.38 & 1.39 & 0.37 & 0.22 & 0.830 & 0.08 \\
\hline & Deaf & 12 & 3.27 & 1.31 & 0.37 & & & \\
\hline \multirow[t]{2}{*}{ VTOV (m/sec) } & Normal-hearing & 14 & $2.70^{*}$ & 0.16 & 0.04 & 3.36 & 0.003 & 1.31 \\
\hline & Deaf & 12 & 2.35 & 0.35 & 0.10 & & & \\
\hline \multirow[t]{2}{*}{$\mathrm{AP}(\mathrm{W})$} & Normal-hearing & 14 & $2,331.50^{*}$ & 408.21 & 109.09 & 3.04 & 0.006 & 1.19 \\
\hline & Deaf & 12 & $1,771.59$ & 531.18 & 153.33 & & & \\
\hline \multirow[t]{2}{*}{$\mathrm{AF}(\mathrm{N})$} & Normal-hearing & 14 & $1,608.00^{* *}$ & 233.46 & 62.39 & 3.14 & 0.001 & 1.22 \\
\hline & Deaf & 12 & $1,329.62$ & 215.13 & 62.10 & & & \\
\hline \multirow[t]{2}{*}{$\mathrm{AV}(\mathrm{m} / \mathrm{sec})$} & Normal-hearing & 14 & $1.50^{*}$ & 0.19 & 0.05 & 1.52 & 0.140 & 0.56 \\
\hline & Deaf & 12 & 1.36 & 0.29 & 0.08 & & & \\
\hline \multirow[t]{2}{*}{$\mathrm{FT}(\mathrm{sec})$} & Normal-hearing & 14 & $0.55^{*}$ & 0.03 & 0.01 & 3.25 & 0.003 & 1.30 \\
\hline & Deaf & 12 & 0.48 & 0.07 & 0.02 & & & \\
\hline
\end{tabular}

JHTOV, jump height from take off velocity; RMP, relative maximal power; ACL, acceleration; VTOV, vertical take off velocity; AP, average power; AF, Average force; AV, average velocity; FT, flight time.

${ }^{*}$ Statistically significant differences $(P \leq 0.05) .{ }^{* *}$ Statistically significant differences $(P \leq 0.01) .{ }^{\dagger}$ Cohen $d$ effect size where $\leq 0.2=$ small, $\leq 0.5=$ medium. and $\leq 0.8=$ large.

priori at an alpha level of 0.05 . Effect sizes $(\dagger)$ are reported based on Cohen recommendations: where $0.2-0.49$ is a small effect, $0.5-0.79$ is a moderate effect, and $\geq 0.8$ is a large effect (Nunes et al., 2021).

\section{RESULTS}

Statistical differences was observed between the groups in all jumping protocols. In the differences detected, normal-hearing athletes are observed to have better scores than deaf athletes in average values. This shows that normal-hearing athletes are clearly in a better condition in the evaluation of the CMJ, SJ, and DJ performances. Findings regarding the comparisons of CMJ, SJ, and DJ performances of deaf and normal-hearing athletes examined in the study are presented in the tables below. CMJ parameters of the athletes and the comparison results between the groups are shown in Table 1.

When the CMJ performances of the athletes were examined in Table 1, a statistical difference was observed between the groups in the JHTOV, RMP, VTOV, AP, AV, and FT parameters ( $P \leq 0.05$; $P \leq 0.01$ only for $\mathrm{AF})$, but there was no statistical difference in ACL $(P>0.05)$. The difference in the JHTOV, RMP, VTOV, AP, $\mathrm{AF}$, and FT parameters was found to be a "large effect," and the difference in the AV parameter was found to be a "moderate effect" according to the "effect size (Cohen $d$ )" classification. SJ parame- ters of the athletes and the comparison results between the groups are shown in Table 2.

When the CMJ performances of the athletes were examined in Table 2, a statistical difference was observed between the groups in the JHTOV, RMP, VTOV, AP, AF, and FT ( $P \leq 0.05$ for AP and AF; $P \leq 0.01$ for JHTOV, RMP, VTOV and FT), but there was no statistical difference in ACL and AV $(P>0.05)$. The difference in the JHTOV, RMP, VTOV, AP, AF, and FT parameters was found to be a "large effect" according to the "effect size (Cohen $d$ )" classification. DJ parameters of the athletes and the comparison results between the groups are shown in Table 3.

When the $\mathrm{CMJ}$ performances of the athletes were examined in Table 3, a statistical difference was observed between the groups in the JHTOV, RMP, ACL, VTOV, and FT ( $P \leq 0.05$ for JHTOV, RMP, ACL, VTOV, and FT) but there was no statistical difference in AP, AF, and AV $(P>0.05)$. The difference in the JHTOV, RMP, VTOV, AP, AF, and FT parameters was found to be a "large effect" according to the "effect size (Cohen $d$ )" classification. Diagrams refering to the billateral CMJ, SJ, and DJ performances (mean values) of the deaf and normal-hearing groups is shown in Fig. 2.

\section{DISCUSSION}

Deaf athletes have an important position among disabled athletes owing to factors such as having athletic performance levels 
Table 2. Comparasion of the athletes by squat jump test

\begin{tabular}{|c|c|c|c|c|c|c|c|c|}
\hline Parameter & Group & No. & $\bar{X}$ & SD & SEM & $t$ & $P$-value & Cohen $d^{t}$ \\
\hline \multirow[t]{2}{*}{ JHTOV (m) } & Normal-hearing & 14 & $0.62^{* *}$ & 0.14 & 0.03 & 4.09 & 0.000 & 1.57 \\
\hline & Deaf & 12 & 0.42 & 0.11 & 0.03 & & & \\
\hline \multirow[t]{2}{*}{ RMP (W/kg) } & Normal-hearing & 14 & $68.06^{* *}$ & 7.79 & 2.08 & 5.42 & 0.000 & 2.20 \\
\hline & Deaf & 12 & 52.54 & 6.63 & 1.91 & & & \\
\hline \multirow[t]{2}{*}{$\mathrm{ACL}\left(\mathrm{m} / \mathrm{sec}^{2}\right)$} & Normal-hearing & 14 & 5.85 & 1.74 & 0.46 & 1.55 & 0.134 & 0.60 \\
\hline & Deaf & 12 & 4.89 & 1.37 & 0.39 & & & \\
\hline \multirow[t]{2}{*}{ VTOV (m/sec) } & Normal-hearing & 14 & $2.79 * *$ & 0.15 & 0.03 & 3.63 & 0.001 & 1.47 \\
\hline & Deaf & 12 & 2.51 & 0.24 & 0.07 & & & \\
\hline \multirow[t]{2}{*}{$\mathrm{AP}(\mathrm{W})$} & Normal-hearing & 14 & $2,040.36^{*}$ & 578.48 & 154.60 & 2.59 & 0.016 & 1.00 \\
\hline & Deaf & 12 & $1,509.48$ & 444.68 & 128.36 & & & \\
\hline \multirow[t]{2}{*}{$\mathrm{AF}(\mathrm{N})$} & Normal-hearing & 14 & $1,340.00^{*}$ & 139.59 & 37.30 & 2.56 & 0.017 & 1.00 \\
\hline & Deaf & 12 & $1,182.60$ & 173.43 & 50.06 & & & \\
\hline \multirow[t]{2}{*}{$\mathrm{AV}(\mathrm{m} / \mathrm{sec})$} & Normal-hearing & 14 & 1.39 & 0.38 & 0.10 & 1.58 & 0.127 & 0.61 \\
\hline & Deaf & 12 & 1.19 & 0.24 & 0.07 & & & \\
\hline \multirow[t]{2}{*}{$\mathrm{FT}(\mathrm{sec})$} & Normal-hearing & 14 & $0.57^{* *}$ & 0.03 & 0.01 & 3.67 & 0.001 & 1.44 \\
\hline & Deaf & 12 & 0.51 & 0.05 & 0.01 & & & \\
\hline
\end{tabular}

JHTOV, jump height from take off velocity; RMP, relative maximal power; $A C L$, acceleration; VTOV, vertical take off velocity; $A P$, average power; $A F$, Average force; $A V$, average velocity; FT, flight time.

${ }^{*}$ Statistically significant differences $(P \leq 0.05) .{ }^{* *}$ Statistically significant differences $(P \leq 0.01) .{ }^{\dagger}$ Cohen $d$ effect size where $\leq 0.2=$ small, $\leq 0.5=$ medium. and $\leq 0.8=$ large.

Table 3. Comparasion of the athletes by drop jump test

\begin{tabular}{|c|c|c|c|c|c|c|c|c|}
\hline Parameter & Group & No. & $\bar{X}$ & SD & SEM & $t$ & $P$-value & Cohen $d^{t}$ \\
\hline \multirow[t]{2}{*}{ JHTOV (m) } & Normal-hearing & 14 & $0.38^{*}$ & 0.08 & 0.02 & 2.80 & 0.012 & 1.08 \\
\hline & Deaf & 12 & 0.30 & 0.07 & 0.01 & & & \\
\hline \multirow[t]{2}{*}{$\mathrm{RMP}(\mathrm{W} / \mathrm{kg})$} & Normal-hearing & 14 & $53.44^{*}$ & 7.48 & 1.99 & 2.56 & 0.017 & 0.99 \\
\hline & Deaf & 12 & 46.52 & 6.08 & 1.75 & & & \\
\hline \multirow[t]{2}{*}{$\mathrm{ACL}\left(\mathrm{m} / \mathrm{sec}^{2}\right)$} & Normal-hearing & 14 & $6.78^{*}$ & 1.96 & 0.52 & -3.50 & 0.002 & 1.37 \\
\hline & Deaf & 12 & 9.71 & 2.31 & 0.66 & & & \\
\hline \multirow[t]{2}{*}{ VTOV (m/sec) } & Normal-hearing & 14 & $2.75^{*}$ & 0.19 & 0.05 & 2.36 & 0.027 & 0.93 \\
\hline & Deaf & 12 & 2.55 & 0.24 & 0.69 & & & \\
\hline \multirow[t]{2}{*}{$A P(W)$} & Normal-hearing & 14 & 2,357.36 & 298.72 & 79.83 & 1.23 & 0.232 & 0.48 \\
\hline & Deaf & 12 & $2,161.42$ & 504.72 & 145.70 & & & \\
\hline \multirow[t]{2}{*}{$A F(N)$} & Normal-hearing & 14 & $1,619.00$ & 146.74 & 39.21 & 1.21 & 0.238 & 0.48 \\
\hline & Deaf & 12 & 1,518.92 & 266.10 & 76.81 & & & \\
\hline \multirow[t]{2}{*}{$\mathrm{AV}(\mathrm{m} / \mathrm{sec})$} & Normal-hearing & 14 & 1.58 & 0.18 & 0.04 & 0.47 & 0.640 & 0.02 \\
\hline & Deaf & 12 & 1.54 & 0.20 & 0.05 & & & \\
\hline \multirow[t]{2}{*}{$\mathrm{FT}(\mathrm{sec})$} & Normal-hearing & 14 & $0.56^{*}$ & 0.03 & 0.01 & 2.54 & 0.018 & 1.20 \\
\hline & Deaf & 12 & 0.51 & 0.05 & 0.01 & & & \\
\hline
\end{tabular}

JHTOV, jump height from take off velocity; RMP, relative maximal power; ACL, acceleration; VTOV, vertical take off velocity; AP, average power; AF, Average force; AV, average velocity; FT, flight time.

${ }^{*}$ Statistically significant differences $(P \leq 0.05) .{ }^{\dagger}$ Cohen $d$ effect size where $\leq 0.2=$ small, $\leq 0.5=$ medium. and $\leq 0.8=$ large.

close to those of normal athletes in addition to the competitions and sports organizations organized specially for them. It is even known that some talented deaf athletes who have resolved their communication problems are in the same team as normal-hearing athletes (Baranauskas et al., 2020; Kurková et al., 2011). Physical structure and jump performance are considered key factors for success in volleyball, where defensive and offensive actions are combined (Pion et al., 2015). The aim of this study was to examine the differences in bilateral CMJ, SJ, and DJ performances between olympic deaf and elite normal-hearing male volleyball 
Normal-hearing athletes

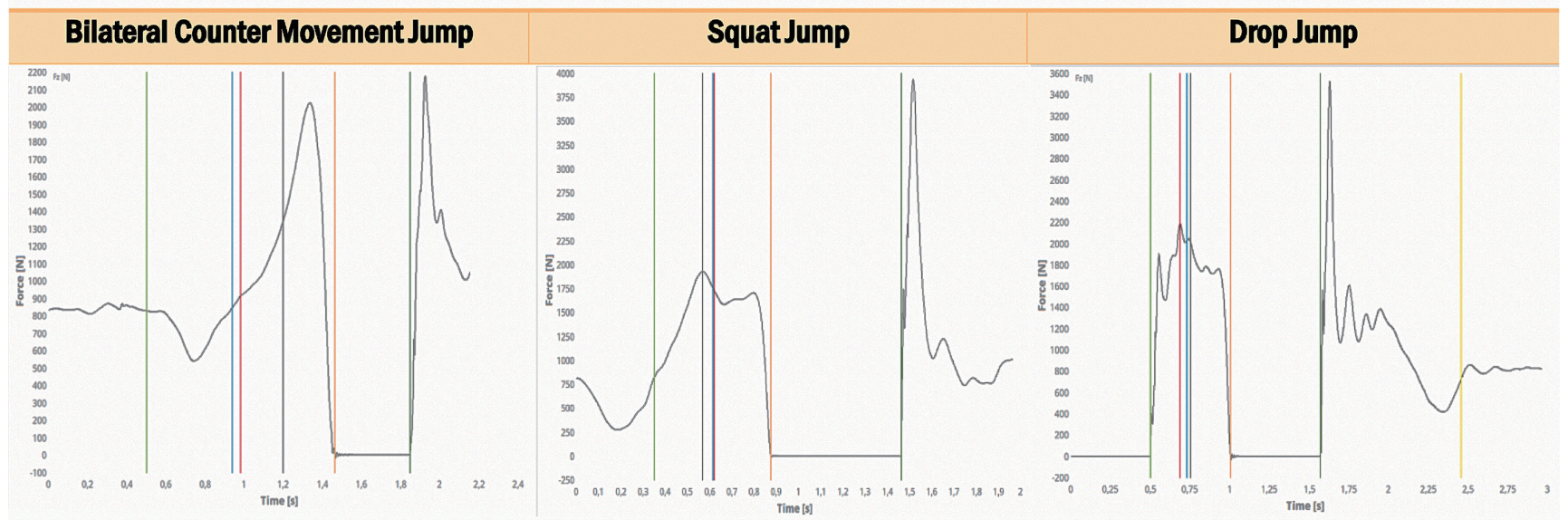

Deaf athletes

Bilateral Counter Movement Jump Squat Jump

Drop Jump
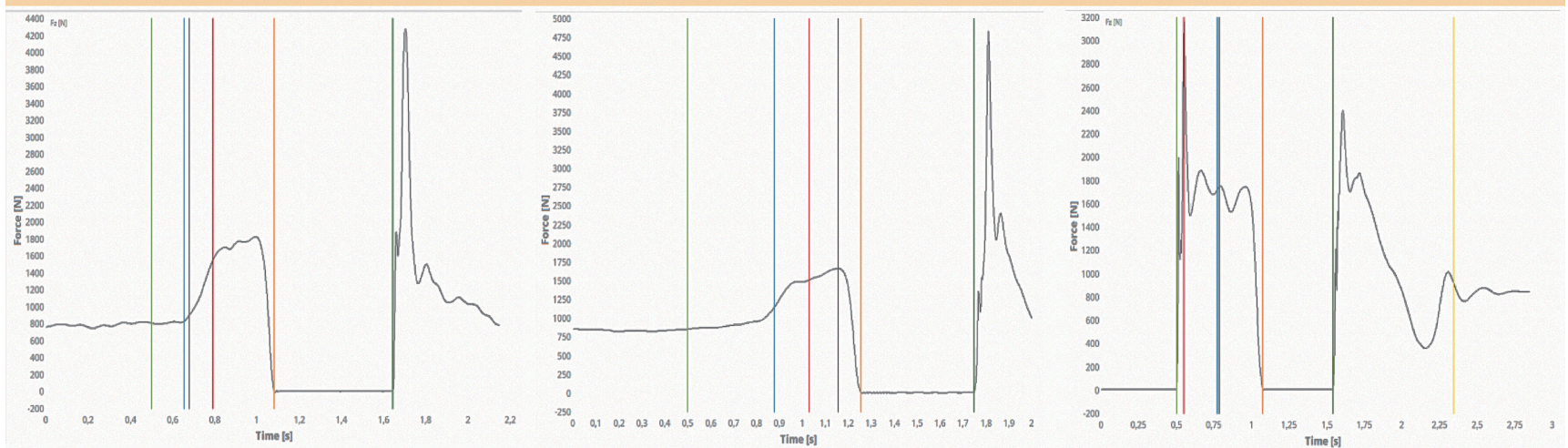

Fig. 2. Representative general jumping performance diagrams from both groups (mean values) during each protocols.

players. The limited study related fitness and athletic characteristics of deaf athletes was observed. To our knowledge, this is the first study to report the jumping performances in different protocols of deaf male volleyball players compared to their normal-hearing peers.

In the present study, when the mechanism of jump protocols applied on the force plate is evaluated, it can be said that CMJ and DJ are methods rather focusing on "stretch shortening cycle" and eccentric-concentric muscle contractions, and SJ concentrates on actions such as explosive power, jumping height, and concentric muscle contraction (Borràs et al., 2011). The main finding in our study is that normal-hearing volleyball players have better jump performances than deaf volleyball players $(P \leq 0.05)$. The existing difference is valid for all three jump protocols (CMJ, SJ, and DJ) (Tables 1-3). In their study comparing the biomechanical characteristics of deaf and normal-hearing female football players, Szulc et al. (2017) stated that normal-hearing football players achieved better results in "spike jump for maximal power" values $(1,828.6 \pm$ 509.4 W and 2,215.2 $\pm 464.5 \mathrm{~W}$, respectively; $P=0.02$, effect size $=0.14$ ). Moreover, normal-hearing athletes were said to have better values in "harmstring/quadriceps ratio" and "maximum voluntary contraction" values. Buśko et al. (2020) reported that deaf female football players had better jump performance than normal-hearing healthy individuals. These results confirm that deaf athletes have lower performance in basic motor features compared to normal athletes.

Peng et al. (2019) investigated the relationship between individually adjusted DJ drop heights and biomechanics. The authors stated that the optimum height adjustment needed to be more than $100 \%$ of the person's CMJ performance (jumping height) to fully detect DJ performance (especially during the landing phase). In other words, the height that a person should use for DJ must be equal to or more than the CMJ performance of the same person. Accordingly, the drop height $(30 \mathrm{~cm})$ used in our study seems to 
be at an acceptable level when associated with the JHTOV values $(0.41 \pm 0.09,0.33 \pm 0.10$, respectively) of the CMJ test obtained from normal-hearing and deaf athletes. Meanwhile, the evaluation of $\mathrm{CMJ}$ and $\mathrm{DJ}$ measurements in deaf and normal-hearing groups in our study makes the results obtained from the two protocols, which resemble each other in terms of jumping mechanism, valuable. Furthermore, when the results of the DJ test in our study were examined (Table 3), the ACL parameter (Tables 2 and 3), which did not differ between the groups in the $\mathrm{CMJ}$ and $\mathrm{SJ}$ tests, exhibited a high statistical difference in the DJ test $(P=0.002$, effect size $=1.37)$, which is an important finding. According to this finding, the lack of performance observed in deaf athletes in the ACL parameter, which refers to acceleration during DJ (maximum vertical jump immediately after the landing), is an issue that needs to be addressed.

Considering that the number of studies investigating the athletic performance (particularly jumping) levels of deaf athletes is limited, it is assumed that it will be a correct method to evaluate the physiological adaptation in the body when interpreting the performance outputs of these athletes. In their studies, Vuillerme et al. (2002) and Śliwowski et al. (2018) emphasized that the best performance of a sportive movement depended on having appropriate postural coordination in the body. In this direction, Trecroci et al. (2015) stated that a jump-based training practice such as "jumping rope" performed in addition to standard football training improves balance and motor performance. Thus, jump-based exercises can be said to be beneficial in minimizing balance problems resulting from vestibular deformation, which is characteristically present in deaf athletes. Schons et al. (2018) reported that an effective jump could be possible with the coordinated action of the muscles and the correct transfer of mechanical energy between the joints. This indicates that there will be major changes in the body's center of gravity in the vertical axis. Postural stability problems, especially in deaf athletes, make it natural to experience problems in points such as the control of the center of gravity and body sway in an action, such as jumping, which takes place in the vertical axis. Accordingly, in the findings of our study, the statistical differences between the groups in favor of normal-hearing athletes in all jump protocols can be said to be important.

When studies in the literature on improving the jumping capacity of volleyball players were reviewed, plyometric (Gjinovci et al., 2017; Mroczek et al., 2017), electromyostimulation (Malatesta et al., 2003) and whole-body vibration (Pérez-Turpin et al., 2014) training practices were found to show improving effects on $\mathrm{CMJ}$ and SJ performances. It is thought that training modules focusing on improving jumping ability can be appropriate in terms of compensating for the loss of jump performance detected in deaf athletes, performing volleyball-specific actions (block, spike, etc.) more effectively, and achieving a performance graph closer to normalhearing volleyball players.

In summary, our results show that a performance difference was determined between the olympic deaf and elite normal-hearing volleyball players. Moreover, the findings of the study performed with the force plate, which is a center of pressure-based measuring device, are considered to be valid and reliable. Therefore, jumping exercises in different different techniques should be included more in the training programs of deaf athletes. Considering that the number of studies on athletic performance in deaf athletes is limited, it can be said that our study will contribute to researchers and coaches in respect of detecting a key ability in volleyball, such as jumping, in different jump protocols.

\section{CONFLICT OF INTEREST}

No potential conflict of interest relevant to this article was reported.

\section{ACKNOWLEDGMENTS}

We would like to thank all the athletes, coaching staff and menagers for their support during the study measuremetns. The authors received no financial support for this article.

\section{REFERENCES}

Akınoğlu B, Kocahan T. Stabilization training versus equilibrium training in karate athletes with deafness. J Exerc Rehabil 2019;15:576-583.

Baranauskas M, Jablonskienė V, Abaravičius JA, Stukas R. Cardiorespiratory fitness and diet quality profile of the Lithuanian team of deaf women's basketball players. Int J Environ Res Public Health 2020;17: 6749 .

Barker LA, Harry JR, Mercer JA. Relationships between countermovement jump ground reaction forces and jump height, reactive strength index, and jump time. J Strength Cond Res 2018;32:248-254.

Beardt BS, McCollum MR, Hinshaw TJ, Layer JS, Wilson MA, Zhu Q, Dai B. Lower-extremity kinematics differed between a controlled dropjump and volleyball-takeoffs. J Appl Biomech 2018;34:327-335.

Borràs X, Balius X, Drobnic F, Galilea P. Vertical jump assessment on volleyball: a follow-up of three seasons of a high-level volleyball team. J Strength Cond Res 2011;25:1686-1694. 
Buśko K, Kopczyńska J, Szulc A. Physical fitness of deaf females. Biomed Hum Kinet 2020;12:101-104.

Coratella G, Beato M, Milanese C, Longo S, Limonta E, Rampichini S, Cè E, Bistonci AV, Schena F, Esposito F. Specific adaptations in performance and muscle architecture after weighted jump-squat vs. body mass squat jump training in recreational soccer players. J Strength Cond Res 2018;32:921-929.

Fuchs PX, Menzel HJK, Guidotti F, Bell J, von Duvillard, SP, Wagner H. Spike jump biomechanics in male versus female elite volleyball players. J Sports Sci 2019;37:2411-2419.

Garcia-Ramos A, Jaric S, Perez-Castilla A, Padial P, Feriche B. Reliability and magnitude of mechanical variables assessed from unconstrained and constrained loaded countermovement jumps. Sports Biomech 2017;16:514-526.

Gjinovci B, Idrizovic K, Uljevic O, Sekulic D. Plyometric training improves sprinting, jumping and throwing capacities of high level female volleyball players better than skill-based conditioning. J Sports Sci Med 2017;16:527-535.

Gonçalves CA, Lopes TJ, Nunes C, Marinho DA, Neiva HP. Neuromuscular jumping performance and upper-body horizontal power of volleyball players. J Strength Cond Res 2021;35:2236-2241.

Kurková P, Válková H, Scheetz N. Factors impacting participation of European elite deaf athletes in sport. J Sports Sci 2011;29:607-618.

Lake J, Mundy P, Comfor P, McMahon JJ, Suchomel TJ, Carden P. Concurrent validity of a portable force plate using vertical jump forcetime characteristics. J Appl Biomech 2018;34:410-413.

Malatesta D, Cattaneo F, Dugnani S, Maffiuletti NA. Effects of electromyostimulation training and volleyball practice on jumping ability. J Strength Cond Res 2003;17:573-579.

McKinley P, Pedotti A. Motor strategies in landing from a jump: the role of skill in task execution. Exp Brain Res 1992;90:427-440.

Mroczek D, Mackala K, Chmura P, Superlak E, Konefal M, Seweryniak T, Borzucka D, Rektor Zbigniew, Chmura, J. Effects of plyometrics training on muscle stiffness changes in male volleyball players. J Strength Cond Res 2019;33:910-921.

Mroczek D, Maćkała K, Kawczynski A, Superlak E, Chmura P, Seweryniak T, Chmura J. Effects of volleyball plyometric intervention program on vertical jumping ability in male volleyball players. J Sports Med Phys Fitness 2017;58:1611-1617.

Nunes AC, Cattuzzo MT, Faigenbaum AD, Mortatti AL. Effects of integrative neuromuscular training and detraining on countermovement jump performance in youth volleyball players. J Strength Cond Res 2021;35:2242-2247.

Peng HT, Song CY, Wallace BJ, Kernozek TW, Wang MH, Wang YH. Effects of relative drop heights of drop jump biomechanics in male vol- leyball players. Int J Sports Med 2019;40:863-870.

Pérez-Castilla A, Rojas FJ, Gómez-Martínez F, García-Ramos A. Vertical jump performance is affected by the velocity and depth of the countermovement. Sports Biomech 2019 Jul 30:1-16. https://doi.org/10.1080 /14763141.2019.1641545. [Epub]

Pérez-Turpin JA, Zmijewski P, Jimenez-Olmedo JM, Jové-Tossi MA, Martínez-Carbonell A, Suárez-Llorca C, Andreu-Cabrera E. Effects of whole body vibration on strength and jumping performance in volleyball and beach volleyball players. Biol Sport 2014;31:239-245.

Pion JA, Fransen J, Deprez DN, Segers VI, Vaeyens R, Philippaerts RM, Lenoir M. Stature and jumping height are required in female volleyball, but motor coordination is a key factor for future elite success. J Strength Cond Res 2015;29:1480-1485.

Psycharakis SG, Eagle SR, Moir GL, Rawcliffe A, Mckenzie C, Graham SM, Lamont HS, Connaboy C. Effects of additional load on the occurrence of bilateral deficit in counter-movement and squat jumps. Res $Q$ Exerc Sport 2019;90:461-469.

Schons P, Da Rosa RG, Fischer G, Berriel GP, Fritsch CG, Nakamura FY, Baroni BM, Peyré-Tartaruga LA. The relationship between strength asymmetries and jumping performance in professional volleyball players. Sports Biomech 2018;18:515-526.

Śliwowski R, Grygorowicz M, Wieczorek A, Jadczak Ł. The relationship between jumping performance. isokinetic strength and dynamic postural control in elite youth soccer players. J Sports Med Phys Fitness 2018;58:1226-1233.

Suarez-Arrones L, Gonzalo-Skok O, Carrasquilla I, Asián-Clemente J, Santalla A, Lara-Lopez P, Núñez FJ. Relationships between change of direction, sprint, jump, and squat power performance. Sports (Basel) 2020;8:38.

Szulc AM, Buśko K, Sandurska E, Kołodziejczyk M. The biomechanical characteristics of elite deaf and hearing female soccer players: comparative analysis. Acta Bioeng Biomech 2017;19:127-133.

Theodorou A, Paradisis G, Panoutsakopoulos V, Smpokos E, Skordilis E, Cooke CB. Performance indices selection for assessing anaerobic power during a 30 second vertical jump test. J Sports Med Phys Fitness 2013;53:596-603.

Tramel W, Lockie RG, Lindsay KG, Dawes JJ. Associations between absolute and relative lower body strength to measures of power and change of direction speed in Division II female volleyball players. Sports (Basel) 2019;7;160.

Trecroci A, Cavaggioni L, Caccia R, Alberti G. Jump rope training: balance and motor coordination in preadolescent soccer players. J Sport Sci Med 2015;14:792-798.

Van Hooren B, Zolotarjova J. The difference between countermovement and squat jump performances: a review of underlying mechanisms 
with practical applications. J Strength Cond Res 2017;31:2011-2020.

Vuillerme N, Nougier V, Teasdale N. Effects of lower limbs muscular fatigue on anticipatory postural adjustments during arm motins in humans. J Sport Sci Med Phy Fit 2002;42:289-294.
Wong TL, Huang CF, Chen PC. Effects of lower extremity muscle fatigue on knee loading during a forward drop jump to a vertical jump in female athletes. J Hum Kinet 2020;72:5-13. 